\title{
Bioactive Peptide Improves Diet-Induced Hepatic Fat Deposition and Hepatocyte Proinflammatory Response in SAMP8 Ageing Mice
}

\author{
Stanley Dumeus ${ }^{\mathrm{a}, \mathrm{b}} \quad$ Marthandam Asokan Shibu ${ }^{\mathrm{a}}$ Wan-Teng Linc Ming-Fu Wang ${ }^{\mathrm{d}}$ \\ Chao-Hung Lai ${ }^{\mathrm{e}}$ Chia-Yao Shen ${ }^{\dagger}$ Yueh-Min Lin ${ }^{g, h}$ Vijaya Padma Viswanadhai \\ Wei-Wen Kuoj Chih-Yang Huanga,k,l \\ a'Graduate Institute of Basic Medical Science, China Medical University, Taichung, Taiwan, ${ }^{\text {bDepartment }}$ \\ of Medicine, State University of Haiti, Faculty of Medicine and Pharmacy, Port-au-Prince, Haiti, \\ 'Department of Hospitality Management, Tunghai University, Taichung, dDepartment of Food and \\ Nutrition, Providence University, Taichung, eDivision of Cardiology, Armed Force Taichung General \\ Hospital, Taichung, fDepartment of Nursing, MeiHo University, Pingtung, Taiwan, פDepartment \\ of Pathology, Changhua Christian Hospital, Changhua, ' $D$ Department of Medical Technology, Jen- \\ Teh Junior College of Medicine, Nursing and Management College, Taipei, Taiwan, 'Department \\ of Biotechnology, Bharathiar University, Coimbatore, India, jDepartment of Biological Science and

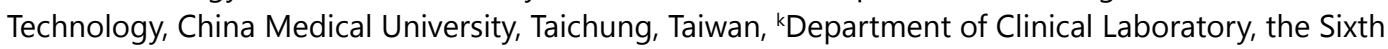 \\ Affiliated Hospital of Guangzhou Medical University, Qingyuan People's Hospital, Guangdong, China, \\ 'Department of Health and Nutrition Biotechnology, Asia University, Taichung, Taiwan
}

\section{Key Words}

Aging - SAMP8 - Hepatosteatosis - NAFLD - Proinflammatory mediators - HFD - Potato protein hydrolysate $\cdot$ Bioactive peptide

\begin{abstract}
Background/Aims: High-fat diet (HFD)-induced nonalcoholic fatty liver disease (NAFLD) poses therapeutic challenges in elderly subjects. Due to lack of efficient drug therapy, plantbased bioactive peptides have been studied as alternative strategy in NAFLD and for less toxicity in elderly. To mimic fatty liver in aging conditions, researchers highly commended the genetically engineered strains SAMP8 (senescence-accelerated mice prone 8). However, there is a paucity of reports about the anti-steatosis effects of bioactive peptides against fatty liver development under a combined action of high-fat diet exposure and aging process. This study was conducted to evaluate the activity of DIKTNKPVIF peptide synthesized from alcalase-generated potato protein hydrolysate (PH), on reducing HFD-driven and steatosisassociated proinflammatory reaction in ageing model. Methods: Five groups of six-month-

W.-W. Kuo and C.-Y. Huang contributed equally to this work.




\section{Cellular Physiology Cell Physiol Biochem 2018;48:1942-1952

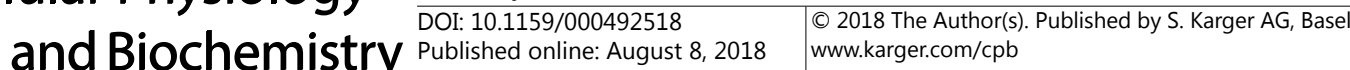 \\ Dumeus et al.: Peptide Ameliorates Hepatic Fat Deposition and Proinflammation in \\ SAMP8}

old SAMP8 mice ( $n=4$, each) were fed either a normal chow (NC group) for 14 weeks upon sacrifice, or induced with a 6-week HFD feeding, then treated without (HCO group) or with an 8-week simultaneous administration of peptide (HPEP group), protein (HPH group) or probucol (HRX group). Liver organs were harvested from each group for histological analysis and immunoblot assay. Results: In contrast to NC, extensive fat accumulation was visualized in the liver slides of $\mathrm{HCO}$. Following the trends of orally administered $\mathrm{PH}$, intraperitoneally injected peptide reduces hepatic fat deposition and causes at protein level, a significant decrease in HFD-induced proinflammatory mediators p-p38 MAPK, FGF-2, TNF- $\alpha$, IL- 6 with concomitant reactivation of AMPK. However, $p$-Foxol and PPAR- $\alpha$ levels were slightly changed. Conclusion: Oral supplementation of $\mathrm{PH}$ and intraperitoneal injection of derived bioactive peptide alleviate proinflammatory reaction associated with hepatosteatosis development in elderly subjects, through activation of AMPK.

(C) 2018 The Author(s)

Published by S. Karger AG, Basel

\section{Introduction}

Aging and high-fat hypernutrition are known as major risk factors for various systemic and organ-specific disorders, including nonalcoholic fatty liver disease (NAFLD) [1]. These combined factors may promote excess fat deposition in liver or steatosis in more than $5 \%$ of hepatic parenchyma, in the context of little amount of alcohol consumption and in absence of existing hepatic pathologies [2]. Hepatosteatosis, the early reversible stage of NAFLD, may progress to nonalcoholic steatohepatitis (NASH) and furtherly to fibrosis, cirrhosis and hepatocellular carcinoma (HCC) [2, 3], with subsequent health and social burden [4]. To study fatty liver and potential therapeutic strategy our lab has successfully developed various dietary murine models and investigated protein hydrolysates from soy or potato with beneficial therapeutic effects [5-7]. Considering special issues with drug administration in elderly, we purposed in this present work to induce NAFLD in SAMP8 specific strains with HFD and evaluate our natural ingredients. Other authors have proposed SAMP8 as the best SAM (senescence-accelerated mice) strains for NAFLD study [8, 9]. Exposing SAMP8 to HFD can properly mimic the disease pathogenesis. In fact, in non-ageing models, our lab's previous animal experiments evidenced that, compared to control group feeding standard chow, young C57BL/6 mice fed a HFD presented abnormal hepatic fat accumulation, increased hepatic level of proinflammatory response [7].

NAFLD progression to NASH (nonalcoholic steatohepatitis) relies greatly on underlying proinflammatory events, which can be fueled by HFD [10, 11]. Exposure to HFD then activates key hepatic proinflammatory mediators such as p38 MAP kinase, TNF- $\alpha$, IL-6 [12], with concomitant impairment of AMPK, a major signaling molecule controlling the pathways of hepatic metabolic homeostasis [13]. Liver-specific re-activation of AMPK suppresses NAFLD onset [13]. AMPK inhibition can be signaled by hepatic inflammatory stimuli [10, 14]. Any therapeutic attempt to decrease inflammatory stimuli in aging liver in conjunction with enhancing or restoring the function of cellular energy sensing regulators such as AMPK could show beneficial effects against NAFLD development. However, despite recent pharmacological advances, there is still no available recommended drug therapy for NAFLD patients, including elderly [15]. However, associated risk factors for NALFD are managed with cholesterol lowering agents or specific drugs for associated diseases, alongside with lifestyle and diet changes $[4,16,17]$. Alternative strategies using plant bioactive compounds were found to retard the development of NAFLD.

Bioactive compounds comprise nature-based ingredients such as nutraceuticals, peptides, antioxidants, with evidence of health-promoting effects via their activities on biological and physicochemical processes such as lipid metabolism control, energy expenditure $[18,19]$. In dietary experimental models $[7,20,21]$, bioactive peptides from popular food crops, including soy and potato, have been studied for their anti-steatosis effects. Dietary intake of amino acids or peptides was found to modulate important molecular metabolic sensors such as AMPK. For instance, administration of Black soy peptides (BSP, 


\section{Cellular Physiology Cell Physiol Biochem 2018;48:1942-1952

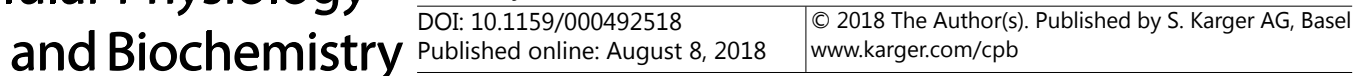 \\ Dumeus et al.: Peptide Ameliorates Hepatic Fat Deposition and Proinflammation in SAMP8}

$10 \%$ ) was able to restore the decreased phospho-AMPK level in white adipose tissue (WAT) of diet-induced obese (DIO) mice fed a HFD [21]. Therefore, considering that HFD-induced inflammation in liver can impair AMPK function related to cellular metabolic homeostasis, we sought to investigate whether our already-tested lipolysis-activating $\mathrm{PH}$ and its derived bioactive peptide [5], by interfering with HFD-driven proinflammatory response, could restore the activity of molecular regulators involving in hepatic lipid metabolism; thus would alleviate NAFLD burden in aging model. On this basis, we have designed a fourteen-week experimental study with SAM8 mice feeding a normal chow diet (NC) or a HFD and then without (HCO) or with simultaneous administration of peptide (HPEP), protein hydrolysate (HPH) or probucol, a hypocholesterolaemic drug (HRX).

We have found that either oral administration of PH or intraperitoneal (i.p.) injection of our peptide reduced lipid droplets pool in SAMP8 mice fed a HFD, in parallel with a significant decrease in protein levels of p-p38 MAPK, FGF-2, TNF- $\alpha$, IL- 6 and reactivation of AMPK. Cumulative data suggest our bioactive peptide as a potential hepato-protective ingredient capable of counteracting hepatosteatosis-related proinflammatory state responsible for NAFLD progression in elderly.

\section{Materials and Methods}

\section{Materials}

The primary antibodies FGF-2 (sc-79), IL-6 (sc-7920), MMP-9 (sc-6841), MMP-2 (sc-13595), PPAR- $\alpha$ (sc-9000), $\alpha$-tubulin (sc-5286), $\beta$-actin (sc-47778), TNF- $\alpha$ (sc-1350) were purchased from Santa Cruz Biotechnology (Santa Cruz, CA, USA); AMPK $\alpha$ (\#2532), p-AMPK $\alpha^{\left(\text {Thr172) }^{2}\right.}$ (\#2535), p-Fox01 (\#9464), p38

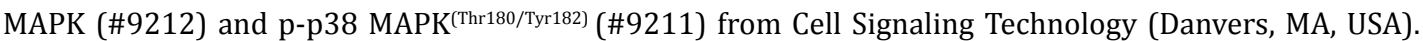
All other reagents and chemicals, including Probucol, were from Sigma Aldrich (St. Louis, MO, USA) unless otherwise mentioned.

\section{Potato-extracted protein hydrolysate and bioactive peptide}

We followed established methods for preparation and purification of potato-extracted Protein Hydrolysate (PH) [4]. Crude potato protein was purchased from Han-Sient Co. (Taipei, Taiwan) and Alcalase from Novo Nordisk A/S (Copenhagen, Denmark). Following the manufacturer's instructions, a reaction mixture was prepared with crude potato protein $(2.5 \%)$ subjected to hydrolysis during $2 \mathrm{~h}$ by alcalase enzyme (equal to $1 \%$ of crude protein weight). An amount of $1 \mathrm{~mL}$ product mixture was obtained from an aqueous combination of protein hydrolysate with o-phthaldialdeyde (OPA) solution, and then incubated for two minutes at room temperature. Then, the product absorbance was obtained by ultraviolet-visible spectrophotometry (Shimazu, UVmini-1240) at wavelength of $340 \mathrm{~nm}$. The product mixture generated a hydrolysate ( $\sim 81 \%$ protein), having a degree of hydrolysis higher than $9.5 \%$ and exhibiting lipolysisstimulating activity on 3T3-L1 cell lines. Different membrane cut-off filters were used to assess the molecular sizes of PH fragments: 55\% of the resultant products weighting less than $1000 \mathrm{Da}, 40 \%$ between 1000 and $6000 \mathrm{Da}$, and only 5\% more than $6000 \mathrm{Da}$.

MB Mission Biotech Co. (Taipei, Taiwan) provided a commercial service for characterization process, including amino acids composition and peptide synthesis. Fractionation work was done by using reverse phase high performance liquid chromatography (RP-HPLC). The resultant fractions were characterized by using tandem mass spectrometry total ion current (MS-MS-TIC) for peptide sequence identification. The ions series for chosen peptides from the MS-MS-TIC chromatogram were analyzed against protein database (UniProt, Solanum tuberosum) by TurboSequest software (Thermo Fisher Scientific, MA, USA). Data analysis revealed DIKTNKPVIF peptide which showed sequence homogeneity with patatin (Accession number Q2MY50) of potato protein. The amino acids (AA) composition of PH and the bioactive peptide is found in Fig. 1.

DIKTNKPVIF peptide was synthesized at the N-terminus and purified by RP-HPLC to 95\% of purity. The synthesis was performed using standard F-moc (9-fluorenylmethoxycarbonyl) chemistry by an ABI 433A, fully automated and programmable Peptide Synthesizer (Applied Biosystems, Foster city, CA, USA), in solid-phase peptide synthesis (SPPS) monitored with the software packages provided with the 


\section{Cellular Physiology Cell Physiol Biochem 2018;48:1942-1952 \begin{tabular}{ll|l} 
and Biochemistry Published online: August 8, 2018 & $\begin{array}{l}\text { (c) } 2018 \text { The Author(s). Published by S. Karger AG, Basel } \\
\text { www.karger.com/cpb }\end{array}$
\end{tabular} \\ Dumeus et al.: Peptide Ameliorates Hepatic Fat Deposition and Proinflammation in SAMP8}

automated equipment. $10 \mathrm{mM}$ of synthetic peptides were dissolved by a solvent made of 1 part of $0.1 \%$ trifluoroacetic acid in double distilled water, and 1 part of $0.1 \%$ trifluoroacetic acid in acetonitrile. The resultant DIKTNKPVIF mixture was aliquoted and kept at $-80^{\circ} \mathrm{C}$ as stock solution.

\section{Animal experiments and treatments}

The Institutional Animal Care and Use Committee (IACUC) of China Medical University (Taichung, Taiwan) approved the animal experiments through IACUC-100-12 protocol. SAMP8 $(\mathrm{n}=20$ ) having 6 months of age were purchased from the National

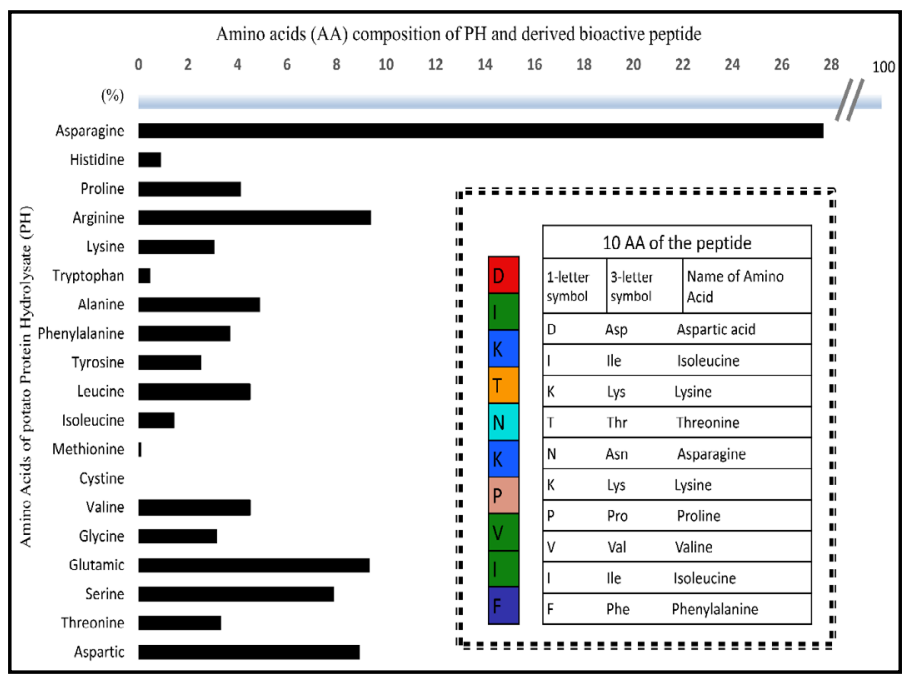

Fig. 1. Amino acids composition of the potato-extracted protein hydrolysate produced by alcalase (PH) and its derived bioactive peptide. Laboratory Animal Centre in Taipei (Taiwan). Mice were individually housed per cage on a 12-hour light-darkness cycle in a room at $22-24{ }^{\circ} \mathrm{C}$ and 45-55 \% humidity. Reverse osmosis treated water was given ad libitum to all groups. NC group fed a normal chow (Rodent Diet 5010 purchased from LabDiet, St. Louis, MO, USA) for 14 weeks upon sacrifice. Remaining groups were induced with a 6-week HFD feeding, then followed without (HCO group) or with an 8-week simultaneous administration of peptide (HPEP group: i.p. $15 \mathrm{mg} / \mathrm{kg} /$ day BW), protein (HPH group: $50 \mathrm{mg} / \mathrm{kg} /$ day BW, oral gavage) or Probucol (HRX group: $500 \mathrm{mg} / \mathrm{kg} /$ day BW, oral gavage). At the end of the experimental period, mice were sacrificed. After overnight fasting, mice were successively anesthetized using ether in a chemical safety hood, euthanized by carbon dioxide asphyxiation, then decapitated. The HFD formula, 58Y1, purchased from LabDiet (St. Louis, MO, USA) consisted of 20.1\% calories from carbohydrates (33\% from sucrose), $18.3 \%$ calories from protein and $60.9 \%$ calories from fat (88\% from lard and $12 \%$ from soybean oil). BSA was used as vehicle. Probucol, a well-known drug, was used in this study as positive control for its role to slow down NASH progression in patients [22].

\section{Masson's trichrome staining (MS)}

Collected hepatic tissues from each group were soaked in $10 \%$ formalin during two weeks, dehydrated by consecutive immersion in alcohols (75\%, 85\%, 90\%, and 100\%, for $5 \mathrm{~min}$ each) and embedded in paraffin wax. Paraffin-embedded tissue blocks were sectioned into $0.2-\mu \mathrm{m}$-thick slides, then deparaffinized by a three-time immersion into xylene for $5 \mathrm{~min}$ each, followed by rehydration with successive soaking in $100 \%, 90 \%, 85 \%$, and 75\% alcohols for 5 min each. Masson's trichrome dye was added for 5 min to tissue slides. Hepatic morphological changes were analyzed and photomicrographs taken with a Zeiss Axiophot microscope.

\section{Tissue protein extraction}

Hepatic tissues of four mice from each group were collected and washed 3 times in phosphate-buffered saline solution. Proper amount of lysis buffer $(100 \mathrm{mg} / \mathrm{mL})$ was added to each sample. The samples were homogenized, positioned on ice for $30 \mathrm{~min}$, followed by centrifugation process with $12,000 \mathrm{rpm}$ for $40 \mathrm{~min}$ at $4{ }^{\circ} \mathrm{C}$. The collected supernatants were stored in microcentrifuge tubes at $-80^{\circ} \mathrm{C}$ for further experiments. The buffer was prepared with $0.02 \mathrm{M}$ Tris, $0.002 \mathrm{M}$ EDTA, $0.05 \mathrm{M}$ 2-mercaptoethanol, $10 \%$ glycerol, phosphatase inhibitor $1 \mu \mathrm{l}$ per $\mathrm{ml}$ and protease inhibitor per $10 \mathrm{ml}$ 


\section{Cellular Physiology Cell Physiol Biochem 2018;48:1942-1952 and Biochemistry \begin{tabular}{l|l} 
DOI: 10.1159/000492518 & $\begin{array}{l}\text { (c) } 2018 \text { The Author(s). Published by S. Karger AG, Basel } \\
\text { www.karger.com/cpb }\end{array}$
\end{tabular} and Biochemistry \\ Dumeus et al.: Peptide Ameliorates Hepatic Fat Deposition and Proinflammation in \\ SAMP8}

Lowry protein assay

Bovine serum albumin (BSA) solution $(0.5 \mathrm{mg} / \mathrm{ml})$ was prepared by mixing proper amount of water with $2 \mathrm{mg} / \mathrm{mL}$ of BSA in a tube. A standard curve was obtained on the basis of ascended grades of protein standards $(0,0.1,0.2,0.3,0.4,0.5 \mathrm{mg} / \mathrm{ml})$ by using the diluted BSA solution $(0.5 \mathrm{mg} / \mathrm{ml})$. In brief, a two-step reaction had taken place. First, the alkaline copper tartrate solution was prepared by mixing proper volume of $\mathrm{Na}_{2} \mathrm{CO}_{3}(2 \% \mathrm{w} / \mathrm{v})$ in $\mathrm{NaOH}(0.1 \mathrm{M})$ with $\mathrm{CuSO}_{4} \cdot 5 \mathrm{H}_{2} \mathrm{O}(1 \% \mathrm{w} / \mathrm{v})$ and $\mathrm{Na}-\mathrm{K}$ tartrate $(1 \% \mathrm{w} / \mathrm{v})$ with a ratio of 98:1:1. A tetradentate complex with four peptide bonds and one central atom of copper was formed in an alkaline medium as resultant of a 10-minute reaction mixture at room temperature (RT) between protein and copper ions $\left(\mathrm{Cu}^{2+}\right.$ and $\left.\mathrm{Cu}^{1+}\right)$ found in the alkaline copper tartrate solution. Second, by adding folin's reagent for a 30-minute reaction at RT, phosphomolybdic-phosphotungstic complex was reduced by $\mathrm{Cu}^{1+}$ ions, with the consequence of generating reduced species characterized by a blue color and an absorbance of 405-750 $\mathrm{nm}$. Afterwards, an Elisa reader was used to determine the OD value at a wavelength of $750 \mathrm{~nm}$.

\section{Western blotting}

Protein product concentrations from liver tissue extracts were assessed by using Lowry protein assay method. Aliquots from each tissue sample were mixed with appropriate amount of $5 \times$ loading dye, then heated for five minutes at $95^{\circ} \mathrm{C}$. The loaded samples were electrophoretically separated in $8 \%, 10 \%$ or $12 \%$ sodium dodecyl sulfate polyacrylamide gel electrophoresis (SDS-PAGE) under constant current of $75 \mathrm{~V}$ from a power source. Afterwards, another sequence of electrophoresis using $50 \mathrm{~V}$ current for $3 \mathrm{~h}$ resulted in proteins transfer to polyvinylidene difluoride (PVDF) membranes (GE Healthcare UK Ltd). Then membranes were incubated with $3 \%$ bovine serum albumin (BSA) in Tris-buffered saline (TBS). Specific primary antibodies (Santa Cruz Biotechnology, Santa Cruz, CA, USA) were added to selected membranes for antigen-antibody reaction. Then, Horseradish peroxidase-labelled secondary antibodies were applied accordingly. Membranes soaking in ECL reagent were photographed with Fujifilm LAS-3000 (GE Healthcare Life sciences). Image J software from NIH (USA) was used for data quantification.

\section{Statistical analysis}

Data shown are expressed as the means \pm SD of three (3) independent experiments. To compare multiple groups, statistical analysis was done by one-way ANOVA based on a general linear model (LSD), using SPSS statistical software (version 12.0). Statistical significance was considered at the level of $\mathrm{p}<0.05$.

\section{Results}

Bioactive peptide administration reduces HFDinduced hepatic fat depot in SAMP8

First, to detect fatty liver development and evaluate the protection effect of $\mathrm{PH}$ and peptide treatments, we examined liver tissue slides from each of the five SAMP8 groups designated as NC, HCO, HPEP, HPH and HRX. Photomicrographs of liver sections stained with Masson's trichrome dye are shown in Fig 2. Compared

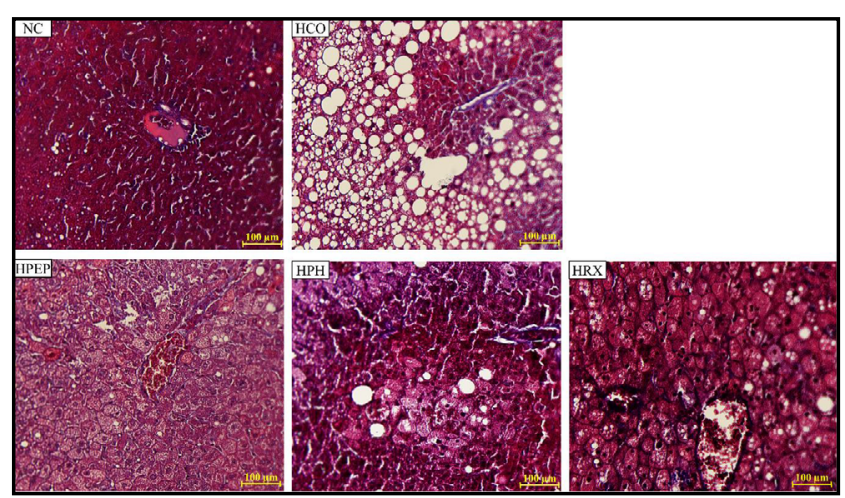

Fig. 2. Peptide and parent protein hydrolysate treatments ameliorate HFD-induced fat depot in SAMP8 mice livers. Histopathological analysis by Masson Trichrome staining (MS) of livers sections from SAMP8 mice. Representative liver tissue slide micrograph from mice fed: a standard chow (NC), a HFD formula as control (HCO), a HFD and treated with indicated dose of DIKTNKPVIF peptide (HPEP), potato protein hydrolysate (HPH) or probucol (HRX). Lipid droplets are few in NC, but widespread micro- and macro- vesicular droplets are displayed in HCO. Their number and size drop considerably in HPEP, HPH and HRX in parallel with amelioration of hepatocytes ballooning. MS stains collagen including vessel walls in blue; cytoplasm, muscles, fibrin, erythrocytes in red, with nuclei in black or dark brown. Images were magnified $\times 400$. The scale bar is $100 \mu \mathrm{m}$. 
to the control group (NC), liver tissue sections from HCO group displayed widespread microvesicular and macrovesicular droplets. In response to the treatments, HFD-induced hepatosteatosis was alleviated in HPEP and HPH groups. Similar to oral supplementation of parent protein hydrolysate, intraperitoneal injection of our peptide obviously decreased the pool and size of fatty droplets in HPEP group. The ballooning degeneration of hepatocytes was also ameliorated in HPEP, HPH and HRX groups, respectively.

DIKTNKPVIF peptide activates hepatic AMPK signaling in order to ameliorate liver steatosis in HDF-fed SAMP8 mice

To investigate important molecular regulators involving in hepatic lipid metabolism, we assessed the protein expression of p-AMPK, PPAR $\alpha$, p-Fox01 (Fig. 3). HFD exposure resulted in a significant inhibition of hepatic AMPK activity in HCO $(\mathrm{P}<0.001)$, contrary to a high presence of AMPK in liver samples from the group fed a standard chow. Surprisingly, HFDinduced impairment of AMPK signaling was not followed by significant decrease in some of its targets such as PPAR $\alpha$ or p-FoxO1. With negligible impact on the aforementioned targets, the treatments in HPEP and HPH significantly reactivate AMPK function which was impaired by HFD exposure. These data suggest not only a direct and intrinsic implication of AMPK in fatty balance in our current model, but a role for our compounds to upregulate this important hepatic metabolic sensor.

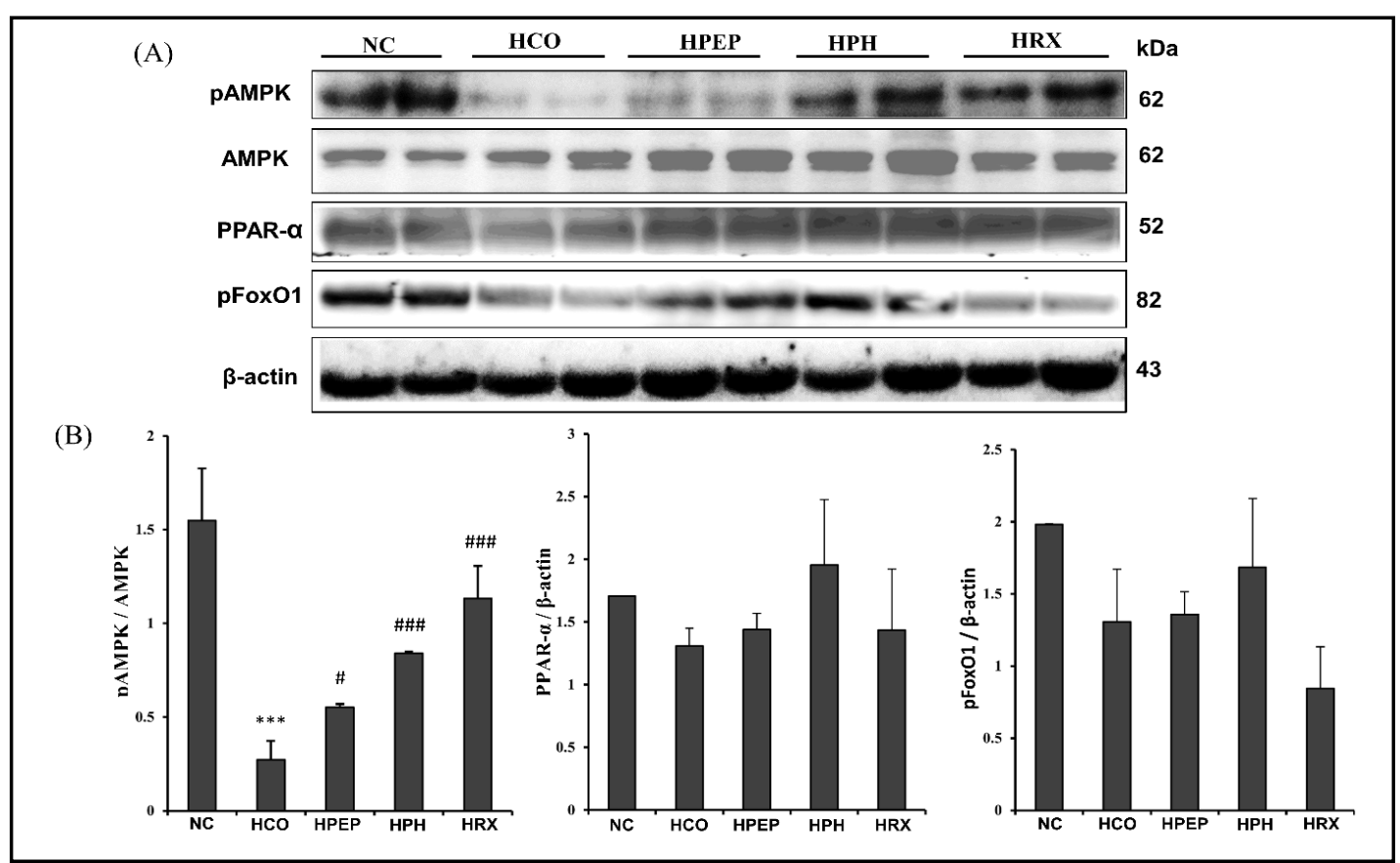

Fig. 3. Peptide and parent protein hydrolysate restore AMPK signaling in order to counteract HFD-driven hepatosteatosis in SAMP8. The expression of AMPK, p-AMPK, p-Fox01, PPAR- $\alpha$ in liver samples from two mice in each group ( $n=4$, per group) fed: a standard chow (NC), a HFD formula as control (HCO), a HFD and treated with indicated dose of DIKTNKPVIF peptide (HPEP), potato protein hydrolysate (HPH) or probucol (HRX) was assessed via Western Blotting. (A). Bar graphs depict densitometric quantification of the Immunoblotting results. (B). $\beta$-actin was used as a loading control. Data are expressed as mean \pm SD of three (3) independent experiments, analyzed by one-way ANOVA and by using SPSS statistical software (version 12.0 ) based on a general linear model (LSD). ${ }^{* * *} \mathrm{P}<0.001$ versus the control group $(\mathrm{NC}) ; \# \mathrm{P}<0.05$, \#\#\#P<0.001 versus the HFD group ( $\mathrm{HCO}$ ). 


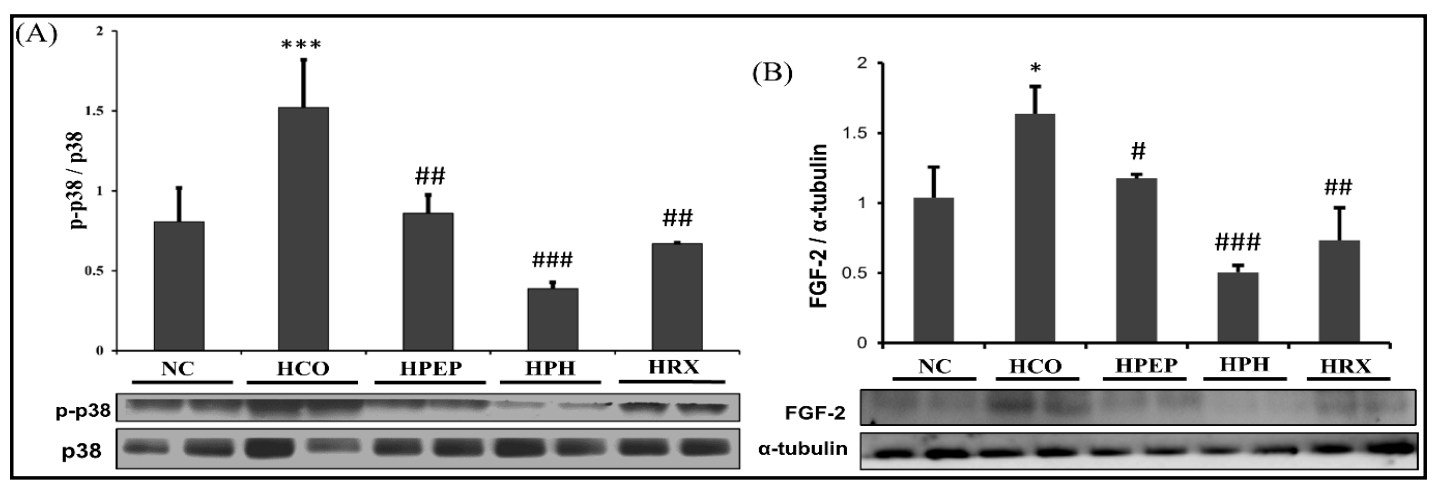

Fig. 4. Effect of potato bioactive peptide and protein hydrolysate on p38 MAPK and FGF-2 activity in SAMP8 fatty liver. The expression of p38, p-p38 (A) and FGF-2 (B) in liver samples from two mice in each group ( $\mathrm{n}=4$, per group) fed: a standard chow (NC), a HFD formula as control (HCO), a HFD and treated with indicated dose of DIKTNKPVIF peptide (HPEP), potato protein hydrolysate (HPH) or probucol (HRX), via Western Blotting. With apposition of bar graphs depicting densitometric quantification of the immunoblotting results. $\alpha$-tubulin was used as a loading control. Data are expressed as mean \pm SD of three (3) independent experiments, analyzed by one-way ANOVA and by using SPSS statistical software (version 12.0) based on a general linear model (LSD). ${ }^{*} \mathrm{P}<0.05,{ }^{* * *} \mathrm{P}<0.001$ versus the control group (NC); \#P<0.05, \#\#P<0.01, $\# \# \# \mathrm{P}<0.001$ versus the HFD group (HCO).

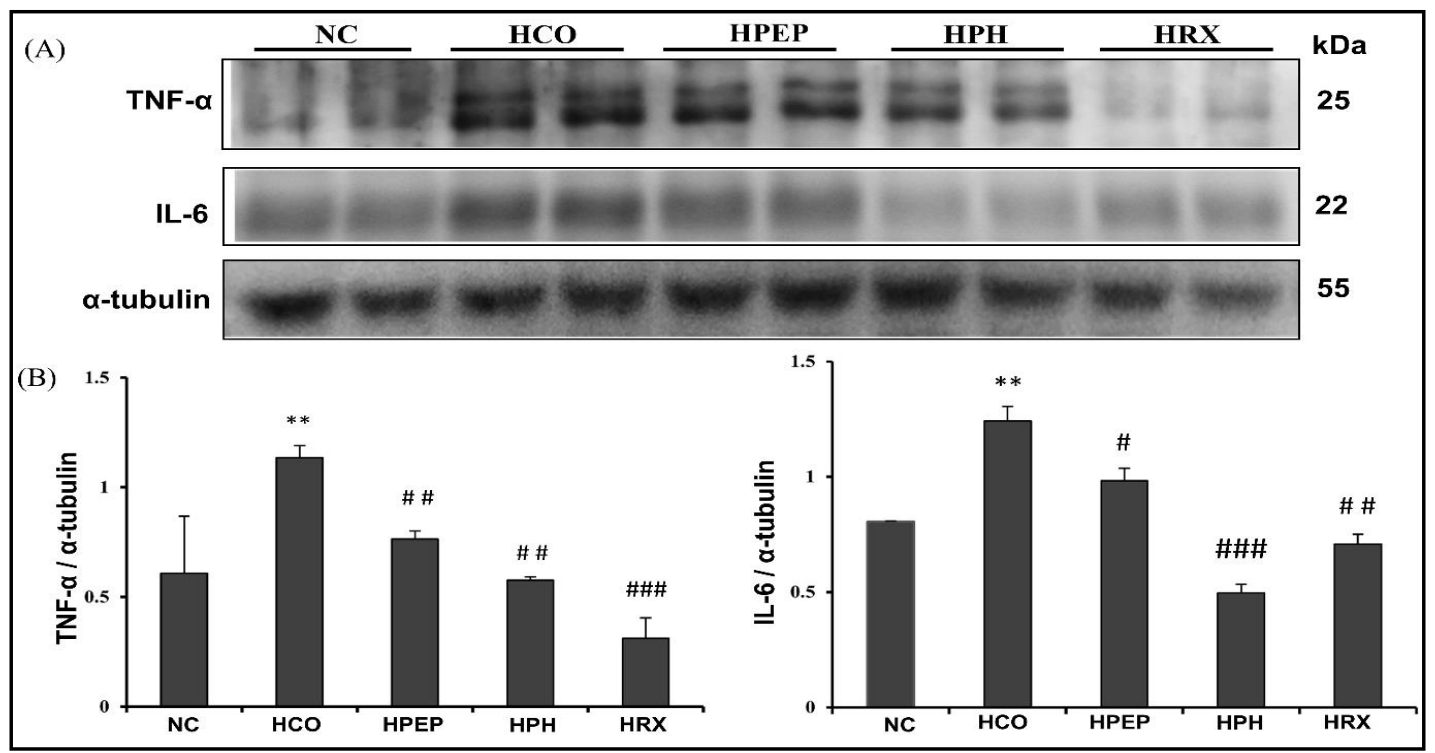

Fig. 5. Effect of potato bioactive peptide and protein hydrolysate on TNF- $\alpha$ and IL- 6 activity in SAMP8 fatty liver. The expression of TNF- $\alpha$ and IL-6 in liver samples from two mice in each group (n=4, per group) fed: a standard chow (NC), a HFD formula as control (HCO), a HFD and treated with indicated dose of DIKTNKPVIF peptide (HPEP), potato protein hydrolysate (HPH) or probucol (HRX) was assessed via Western Blotting (A). Bar graphs depict densitometric quantification of the Immunoblotting results (B). $\alpha$-tubulin was used as a loading control. Data are expressed as mean \pm SD of three (3) independent experiments, analyzed by one-way ANOVA and by using SPSS statistical software (version 12.0) based on a general linear model (LSD). ${ }^{* *} \mathrm{P}<0.01$ versus the control group $(\mathrm{NC})$; $\# \mathrm{P}<0.05$, \#\#P<0.01, \#\#\#P<0.001 versus the HFD group (HCO). 


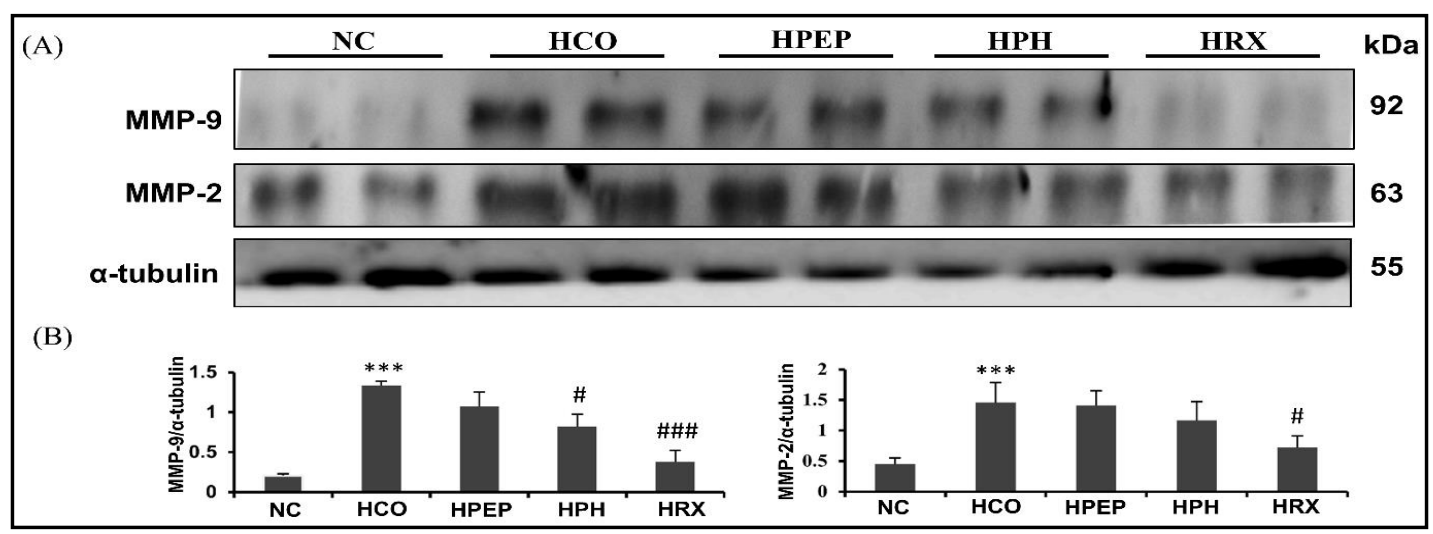

Fig. 6. Effect of potato bioactive peptide and protein hydrolysate on MMP-9 and MMP-2 activity in SAMP8 fatty liver. The expression of MMP-9 and MMP-2 in liver samples from two mice in each group ( $n=4$, per group) fed: a standard chow (NC), a HFD formula as control (HCO), a HFD and treated with indicated dose of DIKTNKPVIF peptide (HPEP), potato protein hydrolysate (HPH) or probucol (HRX) was assessed via Western Blotting (A). Bar graphs depict densitometric quantification of the Immunoblotting results (B). $\alpha$-tubulin was used as a loading control. Data are expressed as mean \pm SD of three (3) independent experiments, analyzed by one-way ANOVA and by using SPSS statistical software (version 12.0) based on a general linear model (LSD). ${ }^{* * *} \mathrm{P}<0.001$ versus the control group (NC); $\# \mathrm{P}<0.05$, \#\#\#P<0.001 versus the HFD group (HCO).

DIKTNKPVIF attenuates proinflammatory reaction in HDF-fed SAMP8 mice through targeting p-p38 MAP kinase

Persistent proinflammatory response fuels fatty liver development and progression. Next, we assessed the expression of main proteins involved in hepatic proinflammatory reaction such as p-p38, FGF-2, TNF- $\alpha$, IL-6, MMP-9 and MMP-2. These aforementioned markers were found to be highly elevated in HCO, compared to NC group. Following the trend of PH supplementation, i.p. injection of our peptide significantly decreased p-p38 $(\mathrm{P}<$ 0.01, HPEP vs HCO) and FGF-2 (Fig. 4), TNF- $\alpha$ and IL-6 (Fig. 5), with moderate reduction in MMP-9 and MMP-2 expression even though both were upregulated by HFD (Fig. 6).

\section{Discussion}

This present study where we investigated DIKTNKPVIF peptide from potato protein hydrolysate aligns with our lab's previous successful work. We have already studied the effects of a bioactive peptide from soy (VHVV) in young mice model of fatty liver [7]. Presently, we have purposed to investigate how effective a potato protein hydrolysate and its derived peptide, a novel compound, can help mitigate NAFLD development in aging model. Following our previous study with a crude protein hydrolysate from potato [6], here we report the results of DIKTNKPVIF peptide synthesized from the original lipolysisactivating $\mathrm{PH}$, highlighting its efficacy to reduce HFD-driven fat deposition and associated proinflammatory response related to NAFLD development in SAMP8 aging mice. Our current model combined two main features: western-like HFD hypernutrition and ageing background. Our present SAMP8 mice model of NAFLD was successfully developed, even though we did not consider measuring and reporting whole body growth parameters or serum and hepatic biochemical indexes such as TG, glucose, adiponectin. The novelty for our present study resides in our attempt for the first time to mimic NAFLD in SAMP8 strains. Previous reports highly recommended senescence-accelerated mice prone 8 as suitable ageing-related model to study NAFLD $[8,9]$. But, to the extent of our current knowledge, no record on HFD-fed SAMP8 mice to study NAFLD is available. However, two recent studies 


\section{Cellular Physiology Cell Physiol Biochem 2018;48:1942-1952

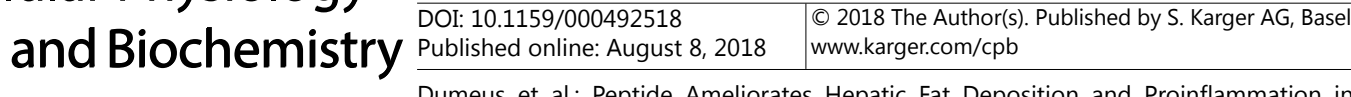 \\ SAMP8}

compared HFD intake with standard chow feeding on hepatic changes in SAMP10) [23, 24]. Their results showed that high-fat diet promoted lipid droplets accumulation in the livers of SAMP10 mice, contrary to normal chow feeding [23]. The same observation was confirmed by histopathological analysis of liver sections from SAMP8 mice groups in our model (Fig. 2).

Steatosis is a consequence of elevated hepatocellular free fatty acids (FFA) pool size created from a disequilibrium between the formation of dietary and de-novo-synthesis FFA, and expenditure through $\beta$-oxidation or export. Resulting lipid droplets are formed from triglyceride (TG) [25-27]. AMPK is a master regulator of both lipid and carbohydrate metabolism in liver [15]. In our study, its function was inhibited by HFD and AMPK became unable to maintain energy balance by itself and by signaling other important targets such as PPAR- $\alpha$ and Fox01 (Fig. 3). However, treatment with our potato extracts significantly upregulated the expression of the phosphorylated form of AMPK, which was drastically dropped by HFD. The same trends were followed with no statistical significance by PPAR- $\alpha, a$ lipid regulator, and by Fox01, a regulator of glucose homeostasis, downstream mediator for insulin signaling (Fig. 3). Therefore, a positive effect of our peptide and protein hydrolysate against NAFLD was by enabling lipid metabolism through activation of AMPK in livers of HPEP and HPH groups, in parallel with alleviating hepatocyte proinflammatory reaction.

In fact, our data show a significant decrease by our peptide in the hepatic protein level of proinflammatory and mitogenic p-p38 and FGF-2 (Fig. 4), of TNF- $\alpha$ and IL-6 (Fig. 5), but with no significant reduction in MMP-9 and MMP-2 (Fig. 6); whereas all these markers were found highly expressed in HCO liver samples. Even though MMP-9 and MMP-2 were highly upregulated in HCO liver samples, their presence denotes rather a proinflammatory response than profibrogenic activity $[28,29]$, because there is no evidence of collagen deposition as revealed by histological data (Fig 2). Above findings are consistent with previous publications from our lab related to VHVV, a bioactive peptide derived from flavourzyme-generated soy protein isolate hydrolysate (F-SPIH) which reduced hepatosteatosis and associated inflammatory events in HFD-induced fatty changes in young C57BL/6 mice. Through i.p. administration, VHVV was able to decrease hepatic inflammatory mediators such as TNF- $\alpha$ and IL-6 [7].

Back to our current work, Western Blot analysis of liver samples indicates that p38 MAPK, another inflammatory activator of TNF- $\alpha$ and IL-6 [30] and well-known clinical biomarker for NAFLD, was found to be highly activated in HCO group, but its activation was impeded by the peptide at almost the same extent as PH (Fig. 4). Together with TNF- $\alpha$ and IL-6 (Fig. 5), the expression of p-p38 indicates a high proinflammatory response in HFD-fed mice, which the peptide administration helped to attenuate. On the other hand, its high expression in HCO liver suggest a mitogenic role in connection with Hepatic Stellate Cells (HSC) activation as result of hepatic lipid deposition [31, 32]. However, contrary to our data and other published work, an inhibitory role to hepatic lipogenesis was reported to be associated with an activation of p38 MAPK signaling pathway [33]. Interestingly, p38 has become a target for drug development [17]. Furthermore, in connection with proinflammatory and mitogenic activity related to HSC activation, a role of the cytokine FGF-2 has been studied [34, 35]. Here we report that our peptide reduced significantly HFD-induced increase of fibroblast growth factor 2 in SAMP8 mice liver (Fig. 4).

In conclusion, the activity of DIKTNKPVIF bioactive peptide and its parent protein $\mathrm{PH}$ in matter of alleviating proinflammatory events underlying HFD-induced hepatic steatosis in elderly was investigated in SAMP8 aging mice feeding a HFD. High-fat diet-promoted changes such as liver steatosis, inflammatory damage, were attenuated by either peptide or parent protein hydrolysate administration which not only enhances lipid metabolism through AMPK activation, but alleviates hepatic proinflammatory response as well. Despite some limitations aforementioned within the discussion section, we conclude that $\mathrm{PH}$ and synthesized biopeptide can be used respectively as hepatoprotective agent for counteracting underlying proinflammatory events responsible for hepatosteatosis development and progression in elderly. 


\section{Cellular Physiology Cell Physiol Biochem 2018;48:1942-1952

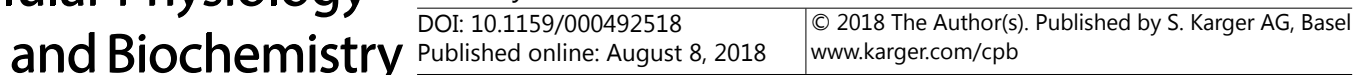 \\ Dumeus et al.: Peptide Ameliorates Hepatic Fat Deposition and Proinflammation in \\ SAMP8}

\section{Acknowledgements}

This study was financially supported in part by grants (MOST-104-2410-H-029-033MY2 and MOST-106-2410-H-029-047-MY2) from the Ministry of Science and Technology of Taiwan and (MOHW106-TDU- B-212- 113004) Taiwan Department of Health, Clinical Trial and Research Center of Excellence. We thank Higie Tamar Dumeus BIEN-AIME for Englishediting assistance. We express our gratitude to Taiwan Ministry of Foreign Affairs (MOFA) Scholarship Program for the Republic of Haiti, through which SD (Faculte de Medecine et de Pharmacie, Universite Etat Haiti) was supported.

\section{Disclosure Statement}

No conflict of interests exists.

\section{References}

1 Kim IH, Xu J, Liu X, Koyama Y, Ma HY, Diggle K, You YH, Schilling JM, Jeste D, Sharma K, Brenner DA, Kisseleva T: Aging increases the susceptibility of hepatic inflammation, liver fibrosis and aging in response to high-fat diet in mice. Age (Dordr) 2016;38:291-302.

-2 European Association for the Study of the Liver (EASL), European Association for the Study of Diabetes (EASD), European Association for the Study of Obesity (EASO): Clinical Practice Guidelines for the management of non-alcoholic fatty liver disease. J Hepatol 2016;64:1388-1402.

-3 Cheng KC, Lin WY, Liu CS, Lin CC, Lai HC, Lai SW: Association of different types of liver disease with demographic and clinical factors. Biomedicine (Taipei) 2016;6:16.

-4 Hsu CS, Kao JH: An update on non-alcoholic fatty liver disease and non-alcoholic steatohepatitis in Asia. Expert Rev Gastroenterol Hepatol 2017;11:759-772.

5 Chiang WD, Huang CY, Paul CR, Lee ZY, Lin WT: Lipolysis stimulating peptides of potato protein hydrolysate effectively suppresses high-fat-diet-induced hepatocyte apoptosis and fibrosis in aging rats. Food Nutr Res 2016;60:31417.

6 Hu WS, Ting WJ, Chiang WD, Pai P, Yeh YL, Chang CH, Lin WT, Huang CY: The Heart Protection Effect of Alcalase Potato Protein Hydrolysate Is through IGF1R-PI3K-Akt Compensatory Reactivation in Aging Rats on High Fat Diets. Int J Mol Sci 2015;16:10158-10172.

7 Chiang WD, Shibu MA, Lee KI, Wu JP, Tsai FJ, Pan LF, Huang CY, Lin WT: Lipolysis-stimulating peptide-VHVV ameliorates high fat diet induced hepatocyte apoptosis and fibrosis. J Funct Foods 2014;11:482-492.

8 Ye X, Meeker HC, Kozlowski PB, Wegiel J, Wang KC, Imaki H, Carp RI: Pathological changes in the liver of a senescence accelerated mouse strain (SAMP8): A mouse model for the study of liver diseases. Histol Histopathol 2004;19:1141-1151.

-9 Vila L, Roglans N, Alegret M, Camins A, Pallas M, Sanchez RM, Vazquez-Carrera M, Laguna JC: Hypertriglyceridemia and hepatic steatosis in senescence-accelerated mouse associate to changes in lipidrelated gene expression. J Gerontol A Biol Sci Med Sci 2007;62:1219-1227.

10 Tilg H, Moschen AR: Evolution of Inflammation in Nonalcoholic Fatty Liver Disease: The Multiple Parallel Hits Hypothesis. Hepatology 2010;52:1836-1846.

11 Hijona E, Hijona L, Arenas JI, Bujanda L: Inflammatory Mediators of Hepatic Steatosis. Mediators Inflamm 2010;2010:7.

12 Choi Y, Abdelmegeed MA, Song BJ: Preventive effects of dietary walnuts on high-fat-induced hepatic fat accumulation, oxidative stress and apoptosis in mice. J Nutr Biochem 2016;38:70-80.

13 Boudaba N, Marion A, Huet C, Pierre R, Viollet B, Foretz M: AMPK Re-Activation Suppresses Hepatic Steatosis but its Downregulation Does Not Promote Fatty Liver Development. EBioMedicine 2018;28:194209.

14 Jung EJ, Kwon SW, Jung BH, Oh SH, Lee BH: Role of the AMPK/SREBP-1 pathway in the development of orotic acid-induced fatty liver. J Lipid Res 2011;52:1617-1625. 
Dumeus et al.: Peptide Ameliorates Hepatic Fat Deposition and Proinflammation in SAMP8

15 Smith BK, Marcinko K, Desjardins EM, Lally JS, Ford RJ, Steinberg GR: Treatment of nonalcoholic fatty liver disease: role of AMPK. Am J Physiol Endocrinol Metab 2016;311:E730-E740.

16 Musso G, Cassader M, Gambino R: Non-alcoholic steatohepatitis: emerging molecular targets and therapeutic strategies. Nat Rev Drug Discov 2016;15:249-274.

17 Singh S, Osna NA, Kharbanda KK: Treatment options for alcoholic and non-alcoholic fatty liver disease: A review. World J Gastroenterol 2017;23:6549-6570.

18 Daliri EB, Oh DH, Lee BH: Bioactive Peptides. Foods 2017;6.

19 Visvanathan R, Jayathilake C, Chaminda Jayawardana B, Liyanage R: Health-beneficial properties of potato and compounds of interest. J Sci Food Agr 2016;96:4850-4860.

-20 Ishiguro K, Kurata R, Shimada Y, Sameshima Y, Kume T: Effects of a sweetpotato protein digest on lipid metabolism in mice administered a high-fat diet. Heliyon 2016;2:e00201.

-21 Jang EH, Moon JS, Ko JH, Ahn CW, Lee HH, Shin JK, Park CS, Kang JH: Novel black soy peptides with antiobesity effects: activation of leptin-like signaling and AMP-activated protein kinase. Int J Obes (Lond) 2008;32:1161-1170.

22 Tokushige K, Hashimoto E, Yatsuji S, Taniai M, Shiratori K: Combined pantethine and probucol therapy for Japanese patients with non-alcoholic steatohepatitis. Hepatol Res 2007;37:872-877.

23 Honma T, Shinohara N, Ito J, Kijima R, Sugawara S, Arai T, Tsuduki T, Ikeda I: High-fat diet intake accelerates aging, increases expression of Hsd11b1, and promotes lipid accumulation in liver of SAMP10 mouse. Biogerontology 2012;13:93-103.

24 Honma T, Yanaka M, Tsuduki T, Ikeda I: Increased Lipid Accumulation in Liver and White Adipose Tissue in Aging in the SAMP10 Mouse. J Nutr Sci Vitaminol (Tokyo) 2011;57:123-129.

25 Donnelly KL, Smith CI, Schwarzenberg SJ, Jessurun J, Boldt MD, Parks EJ: Sources of fatty acids stored in liver and secreted via lipoproteins in patients with nonalcoholic fatty liver disease. J Clin Invest 2005;115:1343-1351.

26 Wu GY, Rui C, Chen JQ, Sho E, Zhan SS, Yuan XW, Ding YT: MicroRNA-122 Inhibits Lipid Droplet Formation and Hepatic Triglyceride Accumulation via Yin Yang 1. Cell Physiol Biochem 2017;44:1651-1664.

-27 Liang YC, Hu JC, Li PY, Huang GJ, Kuo YH, Chao CY: Torenia concolor Lindley var. formosana Yamazaki extracts improve inflammatory response and lipid accumulation via PPARs activation. Biomedicine (Taipei) 2017;7:18.

28 Le NTV, Xue ML, Castelnoble LA, Jackson CJ: The dual personalities of matrix metalloproteinases in inflammation. Front Biosci 2007;12:1475-1487.

29 Parks WC, Wilson CL, Lopez-Boado YS: Matrix metalloproteinases as modulators of inflammation and innate immunity. Nat Rev Immunol 2004;4:617-629.

-30 Guo X, Gerl RE, Schrader JW: Defining the Involvement of p38 $\alpha$ MAPK in the Production of Antiand Proinflammatory Cytokines Using an SB 203580-resistant Form of the Kinase. J Biol Chem 2003;278:22237-22242.

-31 Wobser H, Dorn C, Weiss TS, Amann T, Bollheimer C, Buttner R, Scholmerich J, Hellerbrand C: Lipid accumulation in hepatocytes induces fibrogenic activation of hepatic stellate cells. Cell Res 2009;19:9961005.

32 McKee C, Sigala B, Soeda J, Mouralidarane A, Morgan M, Mazzoccoli G, Rappa F, Cappello F, Cabibi D, Pazienza V, Selden C, Roskams T, Vinciguerra M, Oben JA: Amphiregulin activates human hepatic stellate cells and is upregulated in non alcoholic steatohepatitis. Sci Rep-Uk 2015;5:8812.

-33 Xiong Y, Collins QF, An J, Lupo E, Liu HY, Liu DL, Robidoux J, Liu ZQ Cao WH: p38 mitogen-activated protein kinase plays an inhibitory role in hepatic lipogenesis. J Biol Chem 2007;282:4975-4982.

-34 Schumacher JD, Guo GL: Regulation of Hepatic Stellate Cells and Fibrogenesis by Fibroblast Growth Factors. Biomed Res Int 2016;2016:8323747.

35 Nakamura I, Zakharia K, Banini BA, Mikhail DS, Kim TH, Yang JD, Moser CD, Shaleh HM, Thornburgh SR, Walters I, Roberts LR: Brivanib attenuates hepatic fibrosis in vivo and stellate cell activation in vitro by inhibition of FGF, VEGF and PDGF signaling. PLoS One 2014;9:e92273. 